\title{
Tuning the Hybridization at the Surface of a Heavy-Fermion System
}

\author{
D. V. Vyalikh, ${ }^{1, *}$ S. Danzenbächer, ${ }^{1}$ Yu. Kucherenko, ${ }^{1,2}$ C. Krellner, ${ }^{3}$ C. Geibel, ${ }^{3}$ C. Laubschat,${ }^{1}$ M. Shi, ${ }^{4}$ L. Patthey, ${ }^{4}$ \\ R. Follath, ${ }^{5}$ and S. L. Molodtsov ${ }^{1}$ \\ ${ }^{1}$ Institut für Festkörperphysik, Technische Universität Dresden, D-01062 Dresden, Germany \\ ${ }^{2}$ Institute for Metal Physics, National Academy of Sciences of Ukraine, UA-03142 Kiev, Ukraine \\ ${ }^{3}$ Max-Planck-Institut für Chemische Physik fester Stoffe, Nöthnitzer Strasse 40, D-01187 Dresden, Germany \\ ${ }^{4}$ Swiss Light Source, Paul Scherrer Institute, CH-5232 Villigen-PSI, Switzerland \\ ${ }^{5}$ BESSY GmbH, Albert-Einstein-Strasse 15, D-12489 Berlin, Germany
}

(Received 18 April 2009; published 23 September 2009)

\begin{abstract}
Electron-hybridization phenomena in $\mathrm{YbRh}_{2} \mathrm{Si}_{2}$ were probed by angle-resolved photoemission. It was shown that the $\mathrm{Yb} 4 f-\mathrm{Rh} 4 d$ hybridization strength in the surface region of this heavy-fermion material can be varied by deposition of Ag. Site-specific charge transfer from adatoms leads to change of the energy overlap of the interacting states close to the Fermi energy. Our study demonstrates a new way to tune the hybridization between $4 f$ and valence electrons as well as the induced strong correlation effects at the surface of heavy-fermion systems.
\end{abstract}

DOI: 10.1103/PhysRevLett.103.137601

PACS numbers: 79.60.Dp, 71.27.+a, 73.21.Fg

For almost 30 years, intermetallic rare-earth (RE) materials have attracted considerable attention due to their unusual properties covering magnetism, superconductivity, Kondo, and heavy-fermion (HF) behavior. The key role here plays a partially filled $4 f$ shell that lies mainly inside the ionic core and maintains, therefore, its atomic character and particularly its magnetic moment in solids [1]. Nevertheless, the ground state of RE systems may be strongly affected by increasing interaction between the $4 f$ and delocalized valence states causing screening of the $4 f$ magnetic moments (Kondo effect) or mixed-valent behavior. Thereby, a periodic arrangement of the $4 f$ Kondo impurities (Kondo lattice) leads to HF properties characterized by narrow hybrid bands in momentum $(\boldsymbol{k})$ space that were found in Ce systems like $\mathrm{CeCu}_{2} \mathrm{Si}_{2}$ [2] and $\mathrm{CeSi}_{2}$ as well as $\mathrm{Yb}$ compounds like $\mathrm{YbAl}_{3}$ and $\mathrm{YbRh}_{2} \mathrm{Si}_{2}$ [3].

Recently, a further aspect of HF compounds became a hot topic, namely, the occurrence of quantum critical points (QCPs) [4-9]. In these systems, applying hydrostatic pressure, chemical pressure, or magnetic field leads to a transition from the antiferromagnetic to the paramagnetic HF state. In many systems, the Néel temperature $\left(T_{N}\right)$ is continuously suppressed to $T=0 \mathrm{~K}$ leading to a QCP, where quantum fluctuations modify the metallic properties, opening the door to superconductivity and other novel states of matter [10-12].

In this respect, particular attention was paid to the family of $\mathrm{Yb}$-based compounds [3]. It was found that the nominally isoelectronic materials $\mathrm{YbRh}_{2} \mathrm{Si}_{2}$ and $\mathrm{YbIr}_{2} \mathrm{Si}_{2}$ lie on opposite sides of a QCP. While $\mathrm{YbRh}_{2} \mathrm{Si}_{2}$ orders at $T_{N} \sim$ $70 \mathrm{mK}$, no magnetic ordering was observed in the Ir-based system [13]. At the same time, an increase of the Kondo temperature $\left(T_{K}\right)$ from $\sim 25 \mathrm{~K}\left(\mathrm{YbRh}_{2} \mathrm{Si}_{2}\right)$ to $\sim 40 \mathrm{~K}$ ( $\mathrm{YbIr}_{2} \mathrm{Si}_{2}$ ) shows that the $f-d$ interaction is slightly different in these HF compounds. Substituting Ir for Rh results in a shift of the energy of the valence bands, leading to a change of the energy and $\boldsymbol{k}$ region where the valence and the $f$ states are interacting and thus to a change in the strength of the hybridization [14-17]. Therefore, one can expect that a continuous substitution in the alloy $\mathrm{Yb}\left(\mathrm{Rh}_{1-x} \mathrm{Ir}_{x}\right)_{2} \mathrm{Si}_{2}$ results in a continuous increase of the hybridization and of $T_{K}$, allowing to cross the QCP, which was indeed confirmed [18].

In doped three-dimensional systems, the above correlation phenomena are usually studied by bulk-sensitive transport, specific heat, and magnetization experiments that integrate over large areas in the Brillouin zone and, therefore, do not provide data on the $\boldsymbol{k}$ dependence of the $f$ - $d$ interaction.

On the other hand, the energy position of the interacting states in the surface region can be shifted by charge transfer from adsorbates [19]. Then, in contrast to bulk solids, surface-sensitive angle-resolved photoemission spectroscopy (ARPES) will provide most direct insight into the tuned $f-d$ interaction by probing the $\boldsymbol{k}$-resolved electronic structure of such systems with reduced dimensionalities.

Here, we demonstrate a new possibility to systematically change the energy overlap between the $f$ and $d$ states and in this way to tune and control the hybridization strength in a HF material. We achieved continuous electron doping of $\mathrm{Rh} 4 d$ bands doing a dose deposition of $\mathrm{Ag}$ onto an atomically clean Si-terminated surface of $\mathrm{YbRh}_{2} \mathrm{Si}_{2}$. The ARPES data indicate that the hybridization between the $\mathrm{Yb} 4 f^{13}$ photoemission final state and the valence $\mathrm{Rh} 4 d$ state leads to pronounced dispersion of the $4 f$ originating band. At the $\bar{\Gamma}$ point, a Rh $4 d$ holelike band pushes the $4 f$ band above the Fermi energy $\left(E_{F}\right)$ resulting in a "dent" line shape of the latter. Upon gradual deposition of $\mathrm{Ag}$ metal, the holelike Rh $d$ band moves away from $E_{F}$ causing attenuation of the dent feature. The experimental data were analyzed applying slab band-structure calculations performed by means of a full-relativistic version [20] of the 
linear-muffin-tin-orbital (LMTO) method [21]. Because of well-known failures of LDA-based approaches in description of the strongly localized states in the valence band $(\mathrm{VB})$, the $\mathrm{Yb} 4 f \mathrm{~s}$ were treated as quasicore states.

The experiments were carried out at BESSY (" 13 -ARPES" beam line) and at SLS (SIS beam line). During our measurements performed at a temperature $20 \mathrm{~K}$ (below bulk $T_{K}$ ), the overall energy and angular resolution were set to $15 \mathrm{meV}$ and $0.2^{\circ}$, respectively.

Figure 1(a) shows photoemission (PE) spectrum taken at $h \nu=110 \mathrm{eV}$ when the photoionization cross section of $\mathrm{Yb}$ $4 f$ states is very high, while the ones of $\mathrm{Yb} 5 d$ and $\mathrm{Rh} 4 d$ states are close to Cooper minima [22]. The spectrum exhibits an $\mathrm{Yb} 4 f^{12}$ bulk final-state multiplet between 5.5 and $12 \mathrm{eV}$ binding energy (BE) and two $4 f^{13}$ doublets appear close to $E_{F}$ and at $0.8 \mathrm{eV} \mathrm{BE}$ corresponding to signals from the bulk and the surface, respectively [Fig. 1(a)]. We emphasize that the cleaved surfaces can be populated either by $\mathrm{Yb}$ or by $\mathrm{Si}$ atoms. Note that $\mathrm{Yb}$ termination represents certain difficulties for studying the bulk electronic states near $E_{F}$ and, in particular, the Kondo peak since they are masked by the dominating $4 f$ PE "surface" signal.

An overview band map taken from the Si-terminated surface of $\mathrm{YbRh}_{2} \mathrm{Si}_{2}$ is displayed in Fig. 1(b). The ARPES data were acquired at $45 \mathrm{eV}$ photon energy allowing for both observation of dispersive valence bands and spin-orbit split $\mathrm{Yb} 4 f$ states reflected by sharp horizontal lines just at $E_{F}$ and $1.3 \mathrm{eV}$ BE. In the region close to $E_{F}$, two parabolic

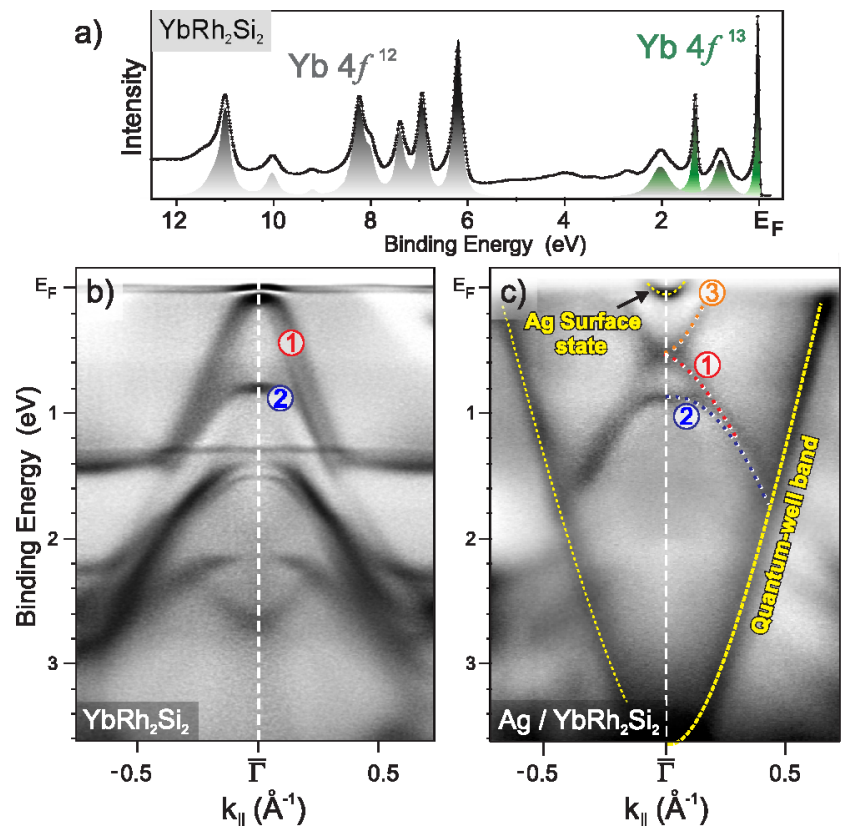

FIG. 1 (color online). (a) Angle-integrated PE spectrum taken from a mainly Si-terminated surface of $\mathrm{YbRh}_{2} \mathrm{Si}_{2}$ at $h \nu=$ $110 \mathrm{eV}$. Experimental ARPES images recorded at $h \nu=45 \mathrm{eV}$ along the $\bar{\Gamma}-\bar{X}$ direction of the $\mathrm{YbRh}_{2} \mathrm{Si}_{2}$ crystal: (b) $\mathrm{Si}$ terminated surface and (c) surface covered by $\sim 1 \mathrm{ML}$ of $\mathrm{Ag}$. Dark areas indicate high PE intensity. holelike bunches of bands [(1) and (2), shown in detail in Fig. 2(a)] are monitored. Band (1), which is split into two subbands with different symmetries, approaches the $E_{F}$ at $\bar{\Gamma}$ and "pushes" the $4 f$ state at $E_{F}$ upwards in energy. The interaction between the $4 f$ and $\mathrm{VB}$ states results in a dentlike shape of the $4 f$ dispersion with obvious crossings the $E_{F}$ at the $\bar{\Gamma}$ point [see blowup in Fig. 2(a)]. This last finding is of high relevance for understanding of the Kondo behavior in RE materials since it is widely believed that the spectral weight in the Kondo-resonance region resides below $E_{F}$ in $\mathrm{Yb}$ systems, in contrast to Ce ones [23]. Our data demonstrate momentum dependence of the spectral function at the $E_{F}$. They show clearly that a lattice of Kondo impurities should be accounted for to make proper description of $\mathrm{YbRh}_{2} \mathrm{Si}_{2}$ compound.

The valence bands calculated for a slab consisting of 15 atomic layers of the sequence shown in Fig. 3(a) are in excellent agreement with the measured photoemission VBs. As follows from the calculations, the bunch of parabolic VBs approaching $E_{F}$ at $\bar{\Gamma}$ is formed mostly by $4 d$ states of Rh atoms in the second slab layer [(1) in Fig. 3(b), rightside panel] and reveals certain $f$ character at the $\mathrm{Yb}$ sites allowing for hybridization with the $\mathrm{Yb} 4 f$ states. The respective bands of bulk $\mathrm{Rh}$ atoms are found at about $0.6 \mathrm{eV} \mathrm{BE}$ [(2) in Fig. 3(b), leftside panel]. This energy position deviates by $\sim 0.2 \mathrm{eV}$ from the value measured experimentally [Fig. 1(b)]. This difference is not astonishing, given the fact that in the calculation, the truncated crystal was simulated by a slab consisting of only a few atomic layers. The energy shift between the bulk and predominantly surface contributions seen in Fig. 3(b) may be related to a reduced charge transfer to Rh subsurface atoms caused by the removal of $\mathrm{Yb}$ surface layer.

Given this assumption is justified, the energy position of the $\mathrm{Rh} 4 d$ bands, which are mainly responsible for the

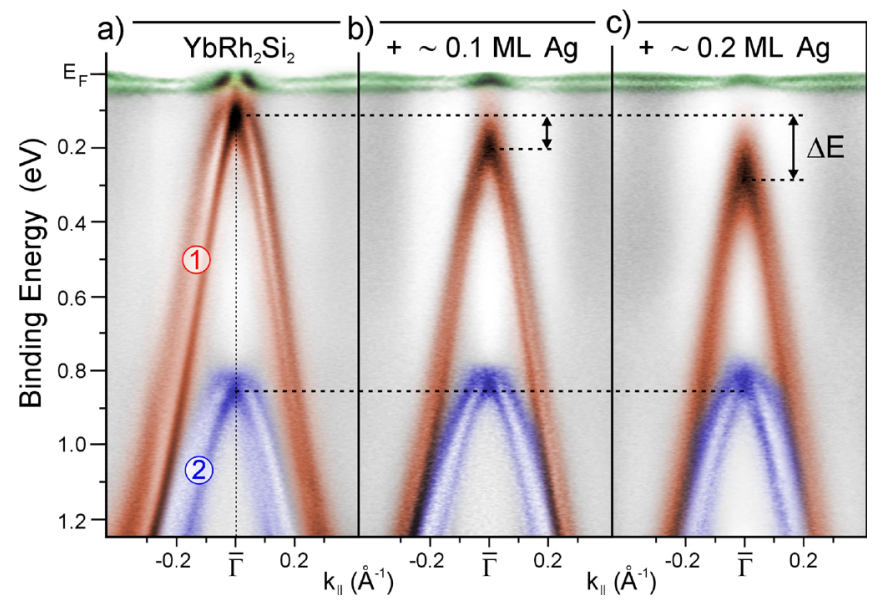

FIG. 2 (color online). Experimental ARPES images taken with $h \nu=45 \mathrm{eV}$ along the $\bar{\Gamma}-\bar{X}$ direction in $\mathrm{YbRh}_{2} \mathrm{Si}_{2}$ for (a) $\mathrm{Si}$ terminated surface and after Ag deposition of (b) $\sim 0.1 \mathrm{ML}$ and (c) $\sim 0.2 \mathrm{ML}$, respectively. High photoemission intensity is shown by dark colors. 


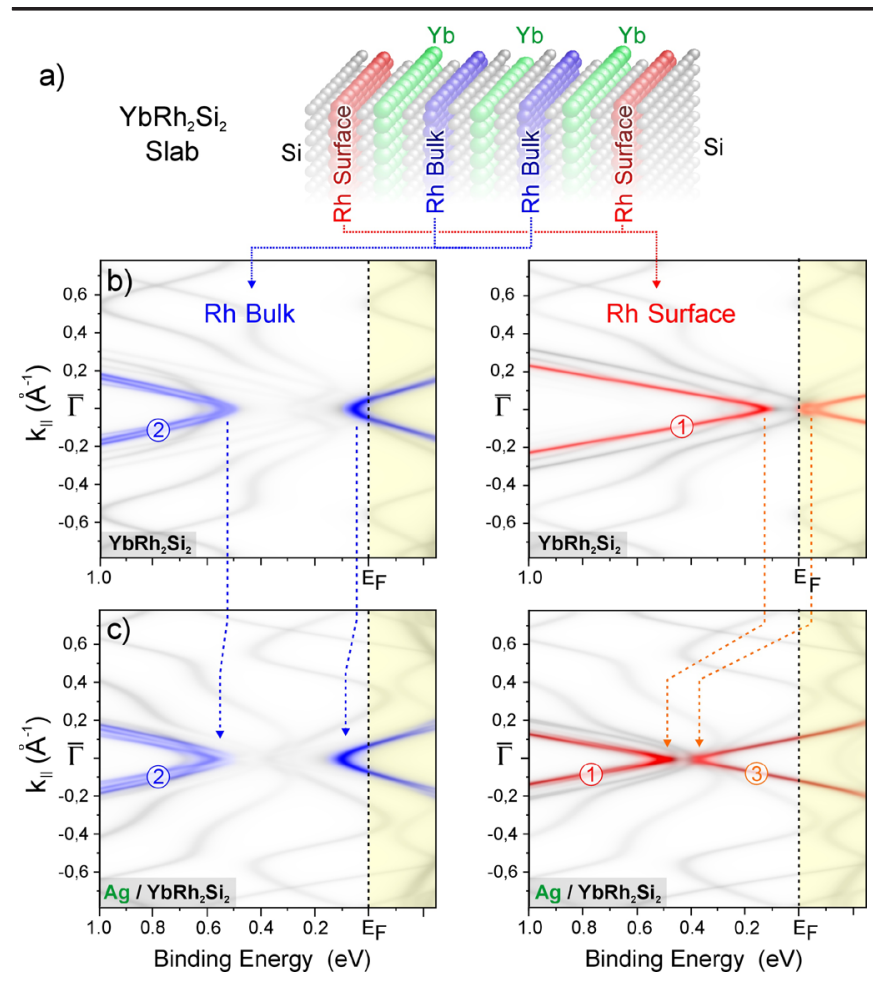

FIG. 3 (color online). (a) Schematic view of the Si-terminated $\mathrm{YbRh}_{2} \mathrm{Si}_{2}$ slab used for the calculations. Valence bands with mainly Rh $4 d$ bulk or surface character calculated for (b) Siterminated slab and (c) the same slab covered on both sides by $1 \mathrm{ML}$ Ag.

interaction with $\mathrm{Yb} 4 f$ states, may heavily be affected in the subsurface region by adsorbates. In this way, the strength of hybridization between $\mathrm{Rh} 4 d$ and $\mathrm{Yb} 4 f$ states that is reflected by the bending of the dent feature at $\bar{\Gamma}$ may be tailored at the subsurface.

The tuning of hybridization may be achieved, for example, by a gradual Ag deposition on the surface. Note, it is important to use for the deposition only Si-terminated samples since the $\mathrm{Yb}$ surface layers would react chemically with the adsorbates, even if noble-metal overlayers are going to be considered. Ag metal has been chosen since it is known to only weakly interact with $\mathrm{Si}$ growing epitaxially on $\mathrm{Si}(100)$ [24]. Additionally, the Ag-derived $4 d$ bands lie at $\mathrm{BE}$ above $3 \mathrm{eV}$ and do not, therefore, interfere with the substrate $\mathrm{Rh} 4 d$ bands.

It is anticipated that the metallic overlayer does not affect the bulk substrate lattice, and, therefore, the chemical pressure on all RE ions in Si-terminated samples remains unchanged upon deposition. However, the electron density in the outermost atomic layers may change due to charge transfer from the adsorbate, and in this way, the topmost $\mathrm{Rh}$ and $\mathrm{Yb}$ layers [second and fourth ones in the slab, respectively, see Fig. 3(a)] may be affected. Particularly, this latter $\mathrm{Yb}$ layer contributes mainly to the $\mathrm{Yb}$ derived PE signal due to small mean-free path of photoelectrons. The expected energy shift of the Rh $4 d$-derived bunch of subsurface bands (1) is confirmed by our calcu- lations. To this end, $\mathrm{Ag}$ monolayers (MLs) were placed on both sides of a 15-layer slab of $\mathrm{YbRh}_{2} \mathrm{Si}_{2}$ assuming regular either $\mathrm{Yb}$ or $\mathrm{Rh}$ positions for the $\mathrm{Ag}$ adatoms. In both cases, similar shifts of the valence bands due to charge transfer of Ag electrons were obtained. Figure 3(c) shows the data calculated for $\mathrm{Ag}$ placed on $\mathrm{Yb}$ sites. The calculations show that the bunch (1) becomes rigidly shifted by about $\sim 0.4 \mathrm{eV}$ towards higher $\mathrm{BE}$ due to charge transfer from $\mathrm{Ag} 5 s$ into $\mathrm{Rh} 4 d$ derived empty states that appear in form of an electronlike band below $E_{F}$ [band (3) in Fig. 3(c)]. In difference to the surface related bunch (1), the bulk bunch (2) is almost unaffected by the doping. The $\mathrm{Ag}$ deposition leads to increasing electron density in the $\mathrm{Si}$ surface and $\mathrm{Rh}$ subsurface atomic layers where the additional charge is distributed mainly between electron states of $p$ and $d$ symmetry around these atoms. The changes in the electron charge reach the fourth atomic layer in the slab increasing slightly occupation of $d$ states around $\mathrm{Yb}$ atoms. The electronic structure of deeper atomic layers remains basically unchanged. The experimental verification of these theoretical predictions can be seen in Fig. 1(c) that presents ARPES spectra taken for $\mathrm{YbRh}_{2} \mathrm{Si}_{2}$ covered with $\sim 1 \mathrm{ML}$ of Ag. The high quality of the samples is demonstrated by formation of a pronounced Ag-derived quantumwell state (QWS) with electronlike parabolic dispersion that superimposes the substrate emission and arrives at the $\bar{\Gamma}$ point at $\sim 3.6 \mathrm{eV} \mathrm{BE}$. This state is not seen in Fig. 3(c), suggesting that the deposited $\mathrm{Ag}$ adlayer structure deviates from the one assumed in our simplified calculations.

A QWS is caused by internal reflections of electron waves both at the Ag surface and at the interface. Since there is no energy gap in the projected substrate band structure, the transmission into the substrate must be symmetry forbidden. We believe therefore that actually $\mathrm{Ag}$ grows as a close-packed layer on the quadratic substrate forming a hexagonally ordered surface isomorphic to $\mathrm{Ag}(111)$. This is the growth mode of $\mathrm{Ag}$ on $\mathrm{Si}(100)$ [24], which is structurally very similar to the Si-terminated $\mathrm{YbRh}_{2} \mathrm{Si}_{2}(001)$. This assumption is supported by the observation of a surface state close to $E_{F}$ at $\bar{\Gamma}$ that is characteristic of the $\operatorname{Ag}(111)$ surface [Fig. 1(c)]. Having other symmetry, this state does not interact with the underlying states of the substrate. The incoherent $\mathrm{Ag} / \mathrm{YbRh}_{2} \mathrm{Si}_{2}(001)$ interface cannot be modeled numerically. Our data show, on the other hand, that the interface is obviously nonreactive and the observed energy shifts are, thus, mainly due to simple charge transfer that should be rather independent from the structural order of the overlayer.

Except the QWS and the surface state, the Rh $4 d$ originating experimental data are in very good agreement with the theoretical predictions: While the bulk-derived substrate bands (2) remain almost unaffected, the Rh $4 d$ subsurface contributions (1) are shifted by $\sim 0.5 \mathrm{eV}$ towards higher BE and the additional parabolic band (3) appears, as predicted, around the $\bar{\Gamma}$ point and approaches the top of the $\mathrm{Rh}$ subsurface band. This shows unambiguously that the 
suggested deposition approach can be used to tune hybridization of the itinerant and more localized electronic states in the surface region of HF systems.

To quantify the hybridization properties in $\mathrm{YbRh}_{2} \mathrm{Si}_{2}$, both the Rh $4 d$ and $\mathrm{Yb} 4 f$ electronic states should be reflected in photoemission. As seen in Fig. 1(c), however, no $4 f$ contribution from the $\mathrm{Yb}$ layers that lie underneath the Rh outermost layers is monitored in the vicinity of $E_{F}$. Obviously, the actual Ag coverage is too thick to experimentally follow hybridization properties in the surface region of $\mathrm{YbRh}_{2} \mathrm{Si}_{2}$. In order to overcome the above obstacle, thinner $\mathrm{Ag}$ coverages were considered. Figure 2 shows ARPES data of $\mathrm{YbRh}_{2} \mathrm{Si}_{2}$ after deposition of $\sim 0.1$ and $\sim 0.2 \mathrm{ML} \mathrm{Ag}$ in comparison to the results measured for the "as cleaved" surface. Similar to $\sim 1$ ML of silver, these thinner coverages reveal the bulk-derived bands, particularly bunch (2), to be unchanged upon Ag deposition. The subsurface derived $\mathrm{Rh} 4 d$ bunch (1), however, is shifted towards higher $\mathrm{BE}$ with increasing $\mathrm{Ag}$ concentration. In contrast to the data depicted in Fig. 1(c), the Yb $4 f$ contributions including the dent feature at $E_{F}$ are clearly seen for both submonolayer coverages.

Moving the Rh $4 d$ states downwards in energy ( $\Delta E$ in Fig. 2), the dispersion of the $4 f$ states is flattened and the bend of the dent feature is reduced. The observed change of dispersion is connected with variations of the effective mass of electrons affecting the HF properties of the system. Simultaneously, the crossings of $E_{F}$ by the $4 f$ state vanish resulting in the conventional scenario for Yb-based materials when the intensity in the Kondo-resonance region lies fully below $E_{F}$. This behavior should certainly be reflected by change of $T_{K}$ in the surface region of $\mathrm{YbRh}_{2} \mathrm{Si}_{2}$ upon deposition of $\mathrm{Ag}$.

On the basis of our ARPES data, which reveal a decrease of the valence-band density between $E_{F}$ and $\sim 0.1 \mathrm{eV} \mathrm{BE}$ with silver coverage, one might expect that $T_{K}$ becomes higher at the $\mathrm{YbRh}_{2} \mathrm{Si}_{2}$ surfaces covered with $\mathrm{Ag}$ [25]. This expectation is in accordance with the corresponding data taken for isoelectronic material $\mathrm{YbIr}_{2} \mathrm{Si}_{2}$ where a similar shift of the $d$ band and, thus, a comparable change of the spectral density close to $E_{F}$ leads to an increase of $T_{K}$ as compared to $\mathrm{YbRh}_{2} \mathrm{Si}_{2}$ [26].

Our study gives experimental insight into the Kondo physics showing that not only states residing directly at $E_{F}$ are responsible for the Kondo behavior including $T_{K}$ value. Valence bands that are located at higher $\mathrm{BE}$, i.e., bands (1) in $\mathrm{YbRh}_{2} \mathrm{Si}_{2}$, can strongly influence this behavior by interaction with the $E_{F}$ states. It would be highly desirable to further prove the suggested scenario by measuring $T_{K}$ in the surface region. The latter, however, is not straightforward for systems with reduced dimensionalities, since the low-energy excitation techniques, which are available for this purpose, are rather bulk sensitive [27].

The discussed mechanism, however, is not restricted to this layered kind of systems and should be valid also for bulk materials. In the latter, the valence bands can be shifted in energy by chemical doping (e.g., substituting
$\mathrm{Rh}$ in $\mathrm{YbRh}_{2} \mathrm{Si}_{2}$ by other transition metals) and the Kondorelated properties can be studied by transport, specific heat, and magnetization experiments.

In summary, our study shows that the electron hybridization can be tuned at the surface of heavy-fermion systems by deposition of adatoms. Thereby, the HF- and Kondo-related surface properties can strongly deviate from the bulk ones. The reported results provide important insight into the mechanism of Kondo behavior and give hints towards further research of bulk materials. At the same time, our approach could open promising perspectives for future studies of $\mathrm{HF}$ and Kondo properties in thin films, multilayers, clusters, or other low-dimensional systems.

This work was supported by the DFG Grant No. VY 64/ 1-1; SFB 463, projects B4, B14, and B16, and by the STCU Grant No. 4930. The authors would like to thank V. Zabolotnyy, A. Varykhalov, A. Kordyuk, and S. Borisenko for their support of our research at the " $1^{3}$-ARPES" beam line of BESSY facility.

*vyalikh@physik.phy.tu-dresden.de

[1] G. R. Stewart, Rev. Mod. Phys. 56, 755 (1984); 73, 797 (2001); 78, 743 (2006).

[2] F. Steglich et al., Phys. Rev. Lett. 43, 1892 (1979).

[3] O. Trovarelli et al., Phys. Rev. Lett. 85, 626 (2000); P. Gegenwart et al., Phys. Rev. Lett. 89, 056402 (2002);

J. Custers et al., Nature (London) 424, 524 (2003); J. Sichelschmidt et al., Phys. Rev. Lett. 91, 156401 (2003).

[4] P. Coleman et al., Nature (London) 433, 226 (2005).

[5] N. D. Mathur et al., Nature (London) 394, 39 (1998).

[6] S. S. Saxena et al., Nature (London) 406, 587 (2000).

[7] O. Stockert et al., Phys. Rev. Lett. 92, 136401 (2004).

[8] H. v. Löhneysen, J. Phys. Condens. Matter 8, 9689 (1996).

[9] H. v. Löhneysen et al., Phys. Rev. Lett. 72, 3262 (1994).

[10] P. Gegenwart et al., Nature Phys. 4, 186 (2008).

[11] D. M. Broun, Nature Phys. 4, 170 (2008).

[12] P. C. Canfield, Nature Phys. 4, 167 (2008).

[13] Z. Hossain et al., Phys. Rev. B 72, 094411 (2005).

[14] D. V. Vyalikh et al., Phys. Rev. Lett. 100, 056402 (2008).

[15] D. V. Vyalikh et al., Phys. Rev. Lett. 96, 026404 (2006).

[16] S. Danzenbächer et al., Phys. Rev. Lett. 96, 106402 (2006).

[17] S. Danzenbächer et al., Phys. Rev. B 75, 045109 (2007).

[18] M. E. Macovei et al., J. Phys. Condens. Matter 20, 505205 (2008).

[19] M. S. Dresselhaus et al., Adv. Phys. 51, 1 (2002).

[20] V. V. Nemoshkalenko et al., Phys. Status Solidi B 120, 283 (1983).

[21] O. K. Andersen, Phys. Rev. B 12, 3060 (1975).

[22] J. J. Yeh and I. Lindau, At. Data Nucl. Data Tables 32, 1 (1985).

[23] F. Reinert et al., Phys. Rev. Lett. 87, 106401 (2001); M. Klein et al., Phys. Rev. Lett. 101, 266404 (2008).

[24] V. A. Gasparov et al., Surf. Sci. 601, 5403 (2007).

[25] Yi.-F. Yang et al., Nature (London) 454, 611 (2008).

[26] S. Danzenbächer et al. (to be published).

[27] M. A. Hossain et al., Nature Phys. 4, 527 (2008). 